\title{
Estudio sobre las necesidades para implementar la gamificación en teleformación
}

Andrea Alcántara Ramírez - Universidad de Granada
Emilio Berrocal de Luna - Universidad de Granada
Juan José Padial Suárez - Universidad de Granada

0000-0002-5176-9552

(D) $0000-0002-3562-8135$

(D) $0000-0003-1960-363 X$

Recepción: 26.06.2021 | Aceptado: 16.10.2021

Correspondencia a través de ORCID: Andrea Alcántara Ramírez

iD 0000-0002-5176-9552

Alcántara, A, Berrocal, E y Padial, JJ (2021). Estudio sobre las necesidades para implementar la gamificación en teleformación. REIDOCREA, 10(30), 36-50.

Resumen: La sociedad y, especialmente, el ámbito de la formación ha experimentado continuos cambios a lo largo de la historia. Estas variaciones han supuesto el surgimiento de nuevos tipos de enseñanza y de nuevas metodologías que además dan la posibilidad de anexión. Este trabajo presenta un análisis de la literatura respecto a esta temática, centrándose en la gamificación como metodología y en la teleformación como tipo de enseñanza. A continuación, se pretende alcanzar una visión más realista y se procede a pasar un cuestionario Ad Hoc tanto a profesorado como alumnado para detectar posibles necesidades a la hora de implementar gamificación en teleformación. Para analizar los datos recogidos se utiliza el programa estadístico SPSS y se procede a analizar las respuestas de cada ítem, las diferentes correlaciones significativas y se lleva a cabo un ANOVA. Por último, tras interpretar los resultados y llegar a una discusión se exponen unas conclusiones generales.

Palabra clave: Gamificación en teleformación

\section{Study on the needs to implement gamification in e-learning.}

Abstract: Society and, especially, the field of training have undergone continuous changes throughout history. These variations have led to the emergence of new types of teaching and new methodologies that also provide the possibility of annexation. This paper presents an analysis of the literature on this topic, focusing on gamification as a methodology and e-learning as a type of teaching. Then, we try to reach a more realistic vision and proceed to pass an Ad Hoc questionnaire to both teachers and students to detect possible needs when implementing gamification in e-learning. To analyse the collected data, the SPSS statistical program is used to analyse the answers of each item, the different significant correlations and an ANOVA is carried out. Finally, after interpreting the results and reaching a discussion, some general conclusions are presented.

Keyword: Gamification in e-learning

\section{Introducción}

La gamificación en el contexto educativo está siendo utilizada tanto para el aprendizaje de ciertos contenidos como para el desarrollo de habilidades y actitudes como comportamientos colaborativos o el estudio autónomo (Caponetto, et al., 2014, citado en Ortiz-Colón, 2018). De hecho, se están empezando a plantear las actividades gamificadas como posibles estrategias didácticas (Area \& Gonzalez, 2015, citado en Ortiz-Colón, 2018). Para que un sistema gamificado sea considerado efectivo en educación, su diseño e implementación debe estar orientado a que sea significativo para el alumno (Domínguez et al, 2013, citado en Covas, 2020). Por ello, Nicholson (2012, citado en Covas, 2020) considera importante que los profesionales integren los elementos centrándolos en los usuarios, es decir, la gamificación debe estar relacionada con las características de los usuarios, como el desarrollo personal, la necesidad de logro, sus creencias, la autoeficacia y las destrezas informáticas, orientándose hacia un mecanismo de juego para que esta experiencia sea relevante 
(Agarwal Prasad, 1999; Kuhlman \& Marshello, 1975; Martocchio \& Webster, 1992; Thiebes et al., 2014; Zweig \& Webster, 2004, citado en Covas, 2020).

La gamificación es una propuesta que se asocia a la educación presencial, asignación de puntos y calificaciones, al fin y al cabo, un sistema de recompensas, pero está empezando a surgir en contextos masivos de enseñanza con el objetivo de mejorar esta y la participación de los miembros (Covas, 2020). Aparicio (2013, citado en Herrera-Sánchez, et al., 2015) señala que el increíble avance de las nuevas tecnologías y de la integración de dispositivos móviles en la sociedad ha permitido crear un escenario donde se pueden introducir técnicas de juegos en diferentes contextos con la finalidad de motivar a los individuos a realizar cualquiera de sus tareas. La combinación de gamificación y tecnologías actuales supone una alternativa interesante que lleva a profesionales y estudiantes a lograr objetivos de aprendizaje con mayor fluidez (Herrera-Sánchez, et al., 2015). También es importante saber cómo enfocar un proyecto gamificado teniendo en cuenta los factores que nos llevan a aplicarlo en una plataforma y sobre qué se va a implementar. (Aparicio et al. 2013, citado en Herrera-Sánchez, et al., 2015).

Prensky (2005, citado en Ortiz-Colón, et al., 2018) contempla que lo que hoy en día busca el alumnado es sentir que sus opiniones tienen valor, seguir sus intereses pudiendo tomar decisiones, trabajar mediante proyectos en grupo utilizando diferentes herramientas, y compartir, cooperar y competir. Por ello, la gamificación es una alternativa que cumple con los requisitos para cumplir estas expectativas pero tiene que diseñarse adecuadamente, lo cual es un reto para los profesionales y aún más dando respuesta a las demandas tecnológicas y digitales actuales.

\section{Objetivos o hipótesis}

El objetivo general de esta investigación es recoger información a través de un cuestionario sobre la percepción que tiene el profesorado y alumnado respecto al uso de gamificación en formación e-learning y detectar posibles necesidades para poder llevarlo a cabo.

A continuación, enumeramos los objetivos específicos que se han perseguido con este estudio:

- Conocer el grado de conocimiento sobre gamificación y el manejo en TIC según el género, rango de edad, rol académico y nivel educativo.

- Conocer cuáles son las plataformas web más utilizadas en formación según los diferentes roles educativos.

- Ver en qué medida la gamificación se considera una buena metodología cuando hay conocimiento sobre su finalidad.

- Conocer el grado de utilidad que consideran que tienen los principales elementos de la gamificación.

- Conocer el nivel de conocimiento y capacitación que tiene el profesorado respecto al manejo en entornos virtuales y recursos digitales.

\section{Método}

Teniendo en cuenta las características expuestas en la presente investigación, se ha llevado a cabo un método descriptivo, el cual permite detallar los escenarios, comportamientos y actitudes de una población de interés seleccionada de forma comprobable (Buendía, et al., 1998, citado en Padial \& Berrocal, 2021) con el fin de 
conocer de manera objetiva las actitudes y comportamientos que presentan tanto alumnado como profesorado de teleformación sobre la gamificación.

Esta investigación se ha planteado entorno a la construcción y aplicación de un instrumento cuantitativo que junto a la revisión bibliográfica sobre los conceptos básicos que se tratan en esta investigación se logra un acercamiento a la realidad que se vive actualmente entorno a estos aspectos y permite analizar y trabajar dichos contenidos de manera eficaz en este trabajo.

\section{Participantes}

La población a la que ha ido destinado este estudio ha sido alumnado y profesorado perteneciente a cualquier nivel educativo, desde Educación Infantil hasta Educación Superior, que hayan impartido o recibido formación e-learning y de distintas edades, desde los 16 años en adelante. La muestra de este estudio finalmente ha sido de $\mathrm{n}=100$ sujetos, seleccionada a través de las respuestas recibidas al cuestionario elaborado y difundido. De esta muestra el $75 \%$ han sido mujeres y el $25 \%$ hombres (gráfico 1), el $69 \%$ estudiantes, el $21 \%$ docentes y el $10 \%$ ambas (gráfico 2 ).

Figura 2. Gráfico "Género"

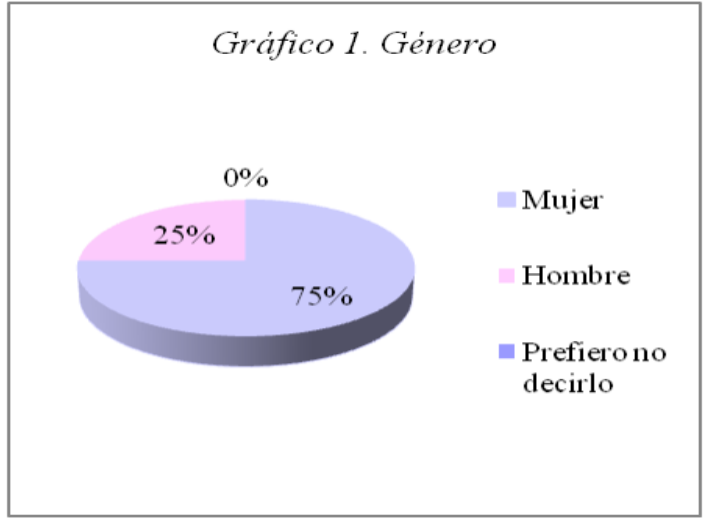

Figura 1. Gráfico "Rol académico"

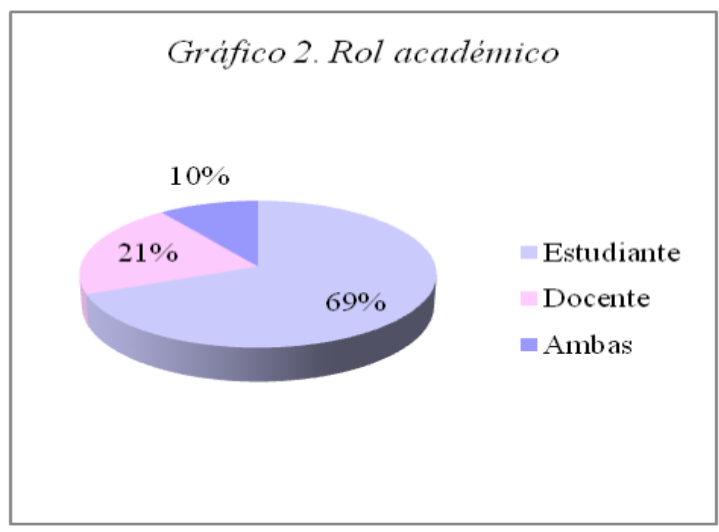

El rango de edad mayoritario de los participantes es de 16 a 25 años (gráfico 3) y los niveles educativos más participativos han sido los Estudios Universitarios con un $69 \%$ y Educación Primaria con un 24\% (gráfico 4).

Figura 3. Gráfico "Edad"

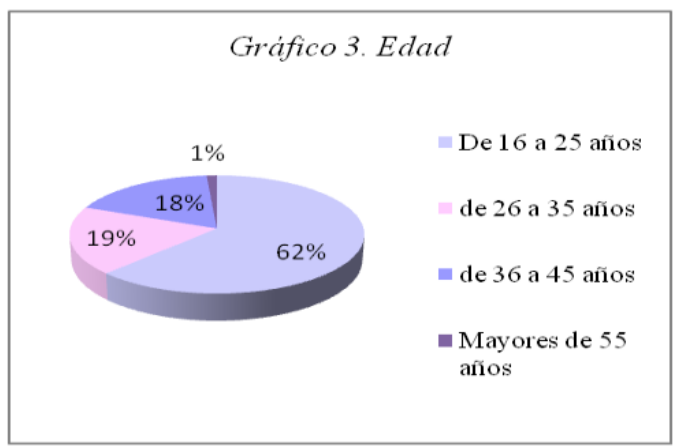

Figura 4. Gráfico "Nivel Educativo"

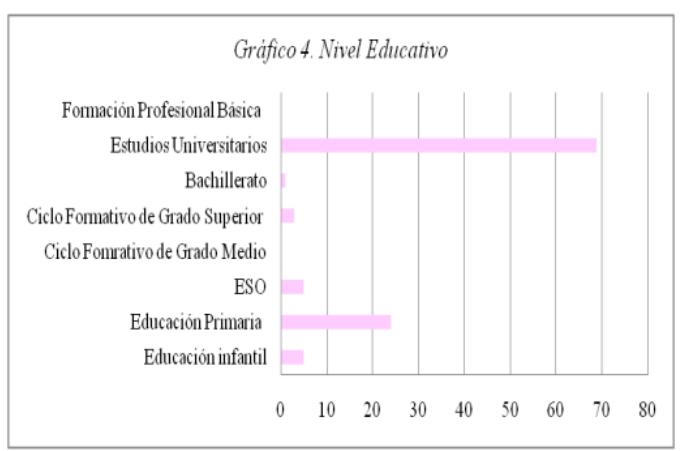

Esta investigación tiene unos principios éticos donde los datos recogidos son de sujetos anónimos y se preservará en todo momento su privacidad e intimidad, respetando la honestidad de la información recolectada (Padial \& Berrocal, 2021). 


\section{Instrumentos}

Para llevar a cabo esta investigación se ha facilitado a la población participante un cuestionario Ad Hoc creado para la ocasión (anexo 1), tratándose de una adaptación de los siguientes cuestionarios: Cuestionario de Satisfacción sobre la gamificación móvil de Arias, et al., (2017), Cuestionario de Satisfacción sobre la aplicación de nuevas metodologías docentes basadas en clase invertida y gamificación a través de Moodle de Francés, et al., (2019), Cuestionario de satisfacción de estudiantes de cursos virtuales de Zambrano (2016) y Cuestionario sobre el grado de conocimiento y capacitación del profesorado participante en las áreas relacionadas con el proceso de autoría de recursos educativos de Romero, et al., (2012). Esta adaptación ha sido sometida a un juicio de expertos, donde, profesionales de diferentes ramas relacionados con la enseñanza y el tema principal de este trabajo, han validado los ítems propuestos para el cuestionario, en claridad, coherencia, redacción y pertinencia mediante una escala Likert 1-5, en la que el valor 1 corresponde a totalmente en desacuerdo y el valor 5 a totalmente de acuerdo. El proceso de validación lo han llevado a cabo tres profesionales: el Coordinador de Formación Continuada del Hospital Universitario de Ceuta, un profesor de la Universidad de Granada y una profesora de la Universidad de Burgos.

Este cuestionario ha sido diseñado para conocer las percepciones del profesorado y alumnado sobre el posible uso de gamificación en la teleformación. Para que resulte útil y sencillo de responder está compuesto por 36 ítems, repartidos en 3 dimensiones. El tipo de respuesta a estas preguntas es tanto de tipo dicotómico (Si/No) como de Escala Likert valorada del 1 al 4, donde 1 corresponde al valor mínimo de acuerdo con el ítem y el 4 el valor máximo.

Este estudio cuenta con una muestra pequeña por lo que este procedimiento de respuesta posibilita interpretar y analizar los datos de forma efectiva. La información recogida durante esta investigación nos permite dar veracidad respecto a lo mostrado anteriormente y, por lo tanto, le da sentido al propósito del estudio (Expósito \& González, 2017). La obtención de datos a través de este cuestionario ha permitido tener una visión más cercana y clara de la realidad sobre las actitudes que se tienen ante la implementación de esta metodología, tanto del profesorado de incorporarla en sus técnicas de enseñanza como del alumnado para recibirla. Por ello, el cuestionario está estructurado en tres dimensiones:

1. La primera parte está dirigida a recopilar los datos personales de los participantes y conocer el punto de partida, es decir, son cuestiones que son determinantes para las respuestas del resto de dimensiones y para los resultados de este estudio.

2. A continuación, se trata de conocer si los elementos, el diseño y la metodología de la gamificación y las tecnologías de la información resultan interesantes, adecuadas y útiles, para introducirlas en la enseñanza.

3. Por último, la tercera parte del cuestionario se ha orientado a conocer la opinión que tienen tanto profesorado como alumnado sobre la capacitación que tienen actualmente los docentes para manejarse en plataformas digitales y para crear recursos didácticos digitales e interactivos.

Con este cuestionario se pretende tener un registro significativo y representativo de la muestra de datos obtenida, por ello se ha utilizado el programa informático SPSS que permite registrar de forma concreta y efectiva la realidad obtenida a través de las respuestas a los cuestionarios rellenados por docentes y alumnos de teleformación, alcanzando así una visión clara de la realidad (Expósito \& González, 2017). 


\section{Procedimiento}

Se han llevado a cabo diferentes acciones mediante las que se han intentado recoger información y conocer diferentes percepciones que se tiene sobre la gamificación, sobre la teleformación y sobre la posibilidad de vincularlas, para ello se ha estructurado la investigación en las siguientes fases:

En primer lugar, se ha realizado un tanteo, es decir, de manera sistemática se ha llevado a cabo una revisión de la literatura sobre el estado de la cuestión. Con ello, se pretendía tener una base fundamentada y teórica sobre los pilares en los que se centra este estudio, la gamificación, la formación y la teleformación.

En la segunda parte de la investigación se ha procedido a pasar un cuestionario a docentes y alumnado, de cualquier nivel educativo, que utilizan diferentes plataformas digitales, con el propósito de obtener información real y actual directamente proporcionada por los usuarios a lo que va dirigida esta investigación y así poder conocer los diferentes conocimientos, actitudes y percepciones, de primera mano, sobre la posibilidad de usar la gamificación en formación e-learning.

Por último, una vez finalizado el procedimiento de recogida de datos, a través del cuestionario, esta información ha sido transferida al programa SPSS para poder analizarla e interpretarla y finalmente establecer los resultados obtenidos.

\section{Resultados}

Como se ha expuesto anteriormente se llevará a cabo un análisis descriptivo con el fin de conocer con exactitud lo recogido a partir del cuestionario. A continuación, se pondrán de manifiesto algunos de los datos obtenidos a lo largo del proceso investigativo con el objetivo de conocer la realidad del profesorado y alumnado entorno a la problemática que se trata en este estudio.

\section{A) Análisis e interpretación individual de cada ítem}

Respecto a este análisis se destacan y comentan algunos de los ítems cuyos datos han sido más significativos para esta investigación:

ÍTEM 9. ¿Considera que la gamificación puede ser una propuesta interesante para implementarla en el ámbito de la formación e-learning?

Figura 5. Gráfico porcentajes “ITEM 9"

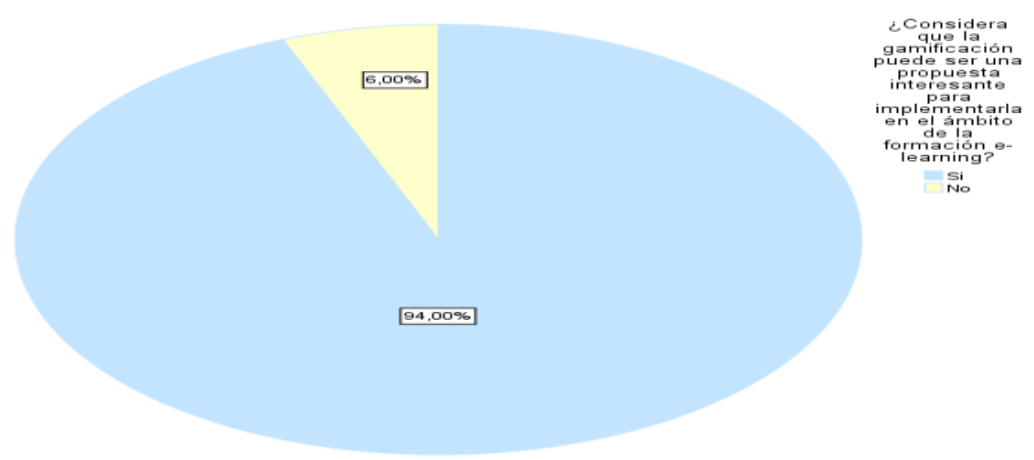


Es importante tener en cuenta que entre el ítem anterior y este había una aclaración sobre lo que era la gamificación dirigida a aquellos participantes que no conocían en qué consistía y en que se basaba esta metodología y así pudiesen reflexionar ante este ítem. Teniendo esto en cuenta, las respuestas reflejan como casi la totalidad de los participantes, 94 sujetos si consideran la gamificación una propuesta interesante para introducirla en formación e-learning y únicamente 6 sujetos no lo consideran como una buena alternativa.

ÍTEM 18. Considero que los recursos didácticos (textos, vídeos, juegos...) permiten avanzar a un ritmo propio de aprendizaje

Figura 6. Media y desviación típica "ÍTEM 18"

\begin{tabular}{|ll|r|}
\hline$N$ & Válidos & 100 \\
& Perdidos & 0 \\
Media & 3,46 \\
Desv. típ. &, 610 \\
\hline
\end{tabular}

Figura 7.Gráfico porcentajes “ÍTEM 18”

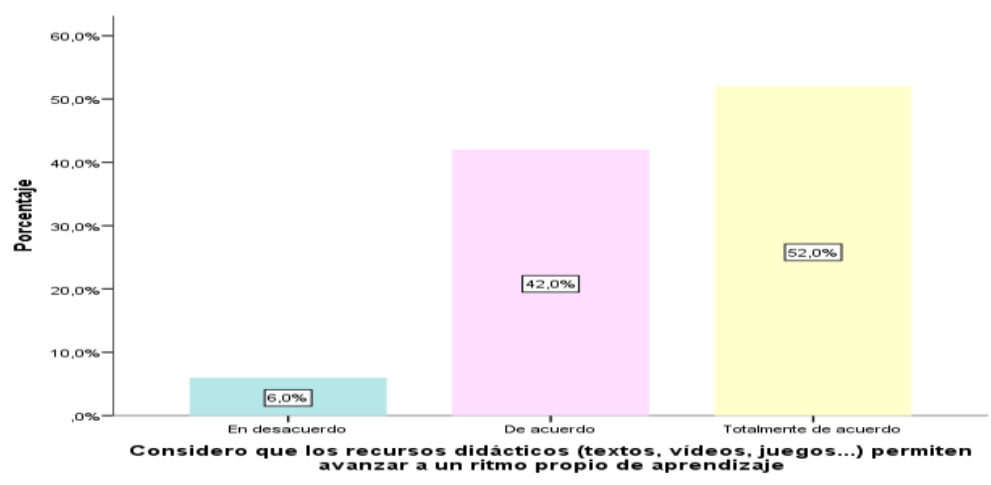

En el gráfico que representa las respuestas a este ítem (figura 7) se puede contemplar como una de las opciones de la escala no ha sido contestada por ningún sujeto, ningún participante está "totalmente en desacuerdo" con que los recursos didácticos permiten avanzar a un propio ritmo de aprendizaje y únicamente 6 sujetos están en desacuerdo y, por lo tanto, los 94 participantes restantes están de acuerdo con la afirmación.

La media es de 3,46 , las respuestas se encuentran entre las opciones "De acuerdo" y "Totalmente de acuerdo" de la escala y la desviación típica es de 0,610, los datos se encuentran bien repartidos y los grupos de respuestas son homogéneos. Con estos valores de media y desviación se puede considerar que el ítem es correcto.

ÍTEM 20. Considero que el desarrollo de actividades en grupo como "retos" mejoran el aprendizaje de un tema

Figura 8. Media y desviación típica "ITTEM 20"

\begin{tabular}{|ll|r|}
\hline N & Válidos & 100 \\
& Perdidos & 0 \\
Media & 3,42 \\
Desv. típ. &, 622 \\
\hline
\end{tabular}


Figura 9. Gráfico porcentajes "ÍTEM 20"

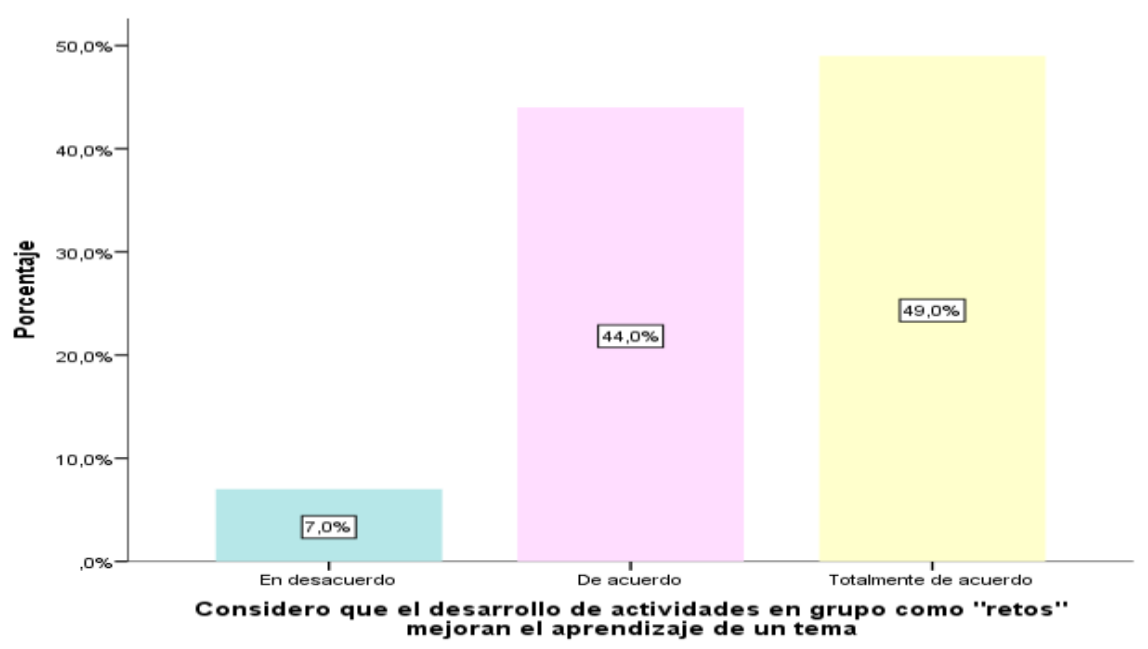

Respecto a este ítem, podemos observar en el gráfico como casi alcanzan la mitad de las respuestas (49) se trata de la opción "Totalmente de acuerdo" y de los participantes restantes 44 sujetos se encuentran "De acuerdo" con la afirmación y únicamente 7 sujetos no consideran que los "retos", otro elemento fundamental de la gamificación, mejoren el aprendizaje de un tema, valor pequeño en comparación con el porcentaje de acuerdo.

En cuanto al valor promedio, es de 3,42 , valor bastante alto, lo que significa que la media de la muestra opina estar entre de acuerdo y totalmente de acuerdo

La desviación de los datos obtenidos en cuanto a la media es de 0,622 , un valor que es pequeño respecto a la escala del cuestionario y por tanto significa que los datos se encuentran bien repartidos y las respuestas son homogéneas. Este ítem cuenta con una media alta y una desviación pequeña por lo que también se trata de un ítem adecuado.

ÍTEM 30. Elaborar animaciones a través del ordenador, con o sin interactividad

Figura 10. Media y desviación típica "ÍTEM 30"

\begin{tabular}{|ll|r|}
\hline$N \quad$ Válidos & 100 \\
& Perdidos & 0 \\
Media & & 2,75 \\
Desv. típ. & 1,009 \\
\hline
\end{tabular}

Figura 11. Gráfico porcentajes "ÍTEM 30"

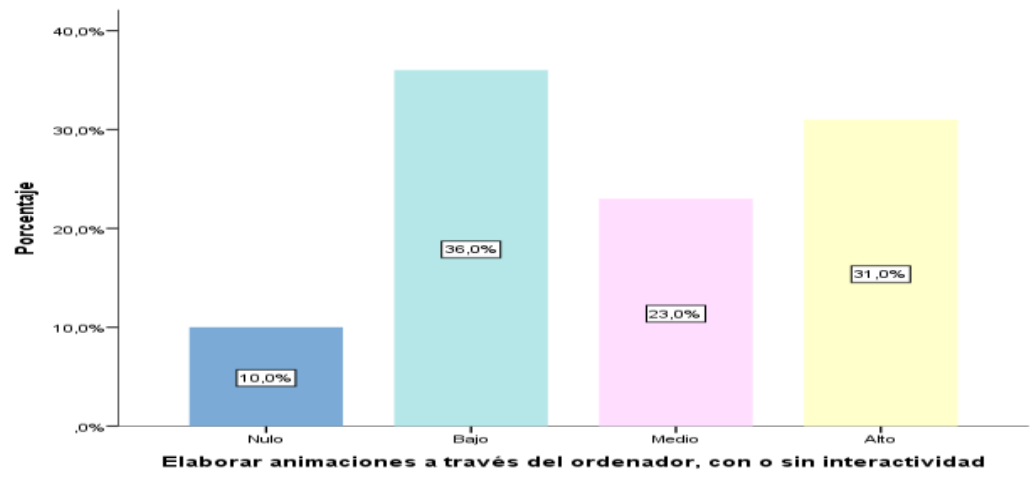


Respecto a la elaboración de animaciones a través del ordenador se encuentran opiniones más repartidas donde un $36 \%$ de la muestra considera que el nivel de conocimiento es bajo, un $31 \%$ que piensa que es alta la capacitación del profesorado, un $23 \%$ que es medio y, por último, 10 sujetos que la consideran nula.

El valor promedio es de 2,75, valor que se encuentra entre el nivel bajo y medio y la desviación típica es de 1,009 por lo que en este caso existe más heterogeneidad entre las respuestas y el ítem debería plantearse de otra manera.

ÍTEM 36. Elaborar actividades con carácter comunicativo mediante las redes sociales

Figura 12. Media y desviación típica "ÍTEM 36"

Figura 13. Gráfico porcentajes "ITEM 36"

\begin{tabular}{|lr|r|}
\hline $\mathrm{N}$ & Válidos & 100 \\
& Perdidos & 0 \\
Media & & 2,75 \\
Desv. típ. &, 903 \\
\hline
\end{tabular}

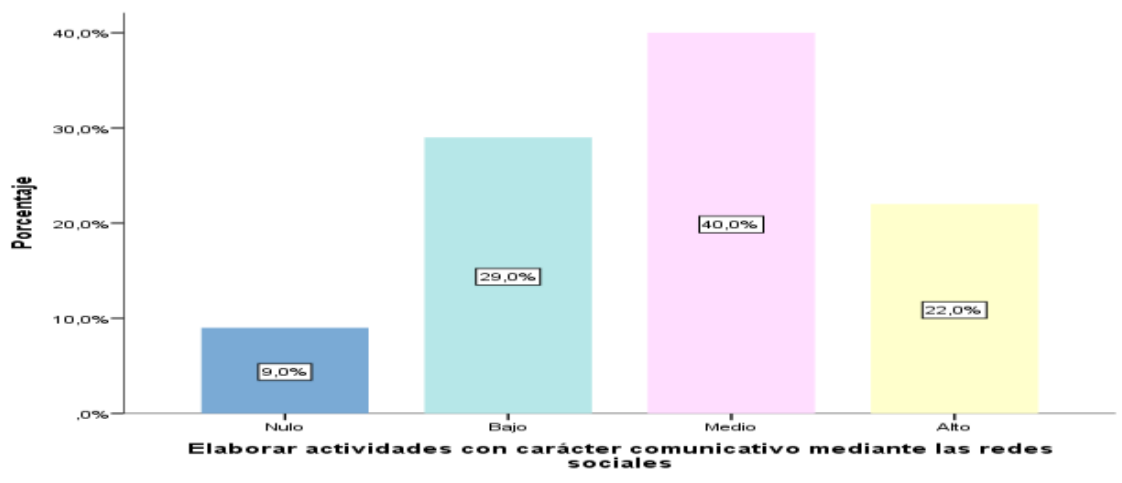

Respecto a la elaboración de actividades con carácter comunicativo a través de redes sociales se encuentran respuestas bastante repartidas, un $40 \%$ considera que el profesorado tiene un nivel de capacitación medio, un $29 \%$ considera que el nivel es bajo, un $22 \%$ considera que los profesores están suficientemente preparados para este aspecto y en contraposición, un 9\% considera que el nivel de conocimientos de los docentes para esta afirmación es nulo.

La media de respuestas a esta afirmación es de 2,75, es decir, de forma general, se puede señalar que la muestra respecto a este ítem considera que el profesorado tiene un nivel de capacitación medio-bajo en elaboración de actividades comunicativas a través de redes sociales. En comparación es un valor bajo y la desviación típica es de 0,903 , que es un valor alto, se puede decir que existe cierta heterogeneidad en las respuestas respecto a este ítem.

\section{B) Análisis e interpretación de las correlaciones entre ítems}

Para conocer la correlación entre los ítems con escala tipo likert del cuestionario, se ha procedido a realizar la Correlación de Pearson ( $r$ ) a través del programa SPSS, donde se debe de tener en cuenta que existe mayor correlación entre los ítems cuanto menor de 0,05 sea el valor de la significación bilateral y cuanto mayor sea el valor de $r$.

A partir de una tabla donde se han correlacionado todos los ítems con todos los ítems se han observado aquellos ítems que son más significativos y se ha creado la Tabla 1 
para relacionar de una manera más clara cada ítem con aquellos otros que tienen mayor correlación. Para ello se ha tenido en cuenta la clasificación propuesta por Ebel (1965) y se han registrado aquellos ítems que tenían un nivel de discriminación muy bueno $(0,4<r)$ y bueno $(0,39<r<0,29)$.

Tabla 1. Mejores correlaciones de los ítems

\begin{tabular}{|c|c|}
\hline ÍTEM 10 & ÍTEMS $12,13,14,31$ Y 36 \\
\hline ÍTEM 11 & ÍTEMS 12,15 y 34 \\
\hline ÍTEM 12 & ÍTEMS 10, 11,13, 14, 19, 20, 24, 26, 31, 32, 34, 36 у 36 \\
\hline ITEM 13 & ÍTEMS $10,12,14,18,20,24,26,31$ y 32 \\
\hline ITTEM 14 & ÍTEMS 10, 12, 13, 16, 25, 26, 31, 32, 33, 34, 35 у 36 \\
\hline ÍTEM 15 & ÍTEM 11 \\
\hline ÍTEM 16 & ÍTEMS $14,17,18,19$, y 20 \\
\hline ITEM 17 & ITEMS 16,20 y 24 \\
\hline ÍTEM 18 & ÍTEMS 12, 13, 16, 19, 20, 21, 22 у 24 \\
\hline ÍTEM 19 & ÍTEMS 12, 18, 20 y 21 \\
\hline ÍTEM 20 & ÍTEMS $12,13,16,17,18,19,21$ y 24 \\
\hline ITTEM 21 & ÍTEMS 18, 19, 20 y 22 \\
\hline ITTEM 22 & ÍTEMS 18, 21 y 23 \\
\hline ÍTEM 23 & ÍTEMS 22, 24 y 26 \\
\hline ÍTEM 24 & ÍTEMS $12,17,18,20,23,26,31,32,35$ y 36 \\
\hline ITEM 25 & ITEMS $14,26,27,28,29,30,31,32,33,34,35$ у 36 \\
\hline ÍTEM 26 & ÍTEMS 12,14, 23, 24, 25, 27, 28, 29, 30, 31, 32, 33, 34, 35 у 36 \\
\hline ITTEM 27 & ÍTEMS 25, 26, 27, 28, 29, 30, 31, 32, 33, 34, 35 у 36 \\
\hline ÍTEM 28 & ÍTEMS $25,26,27,28,29,30,31,32,33,34,35$ y 36 \\
\hline ITTEM 29 & ÍTEMS $25,26,27,28,29,30,31,32,33,34,35$ у 36 \\
\hline ITTEM 30 & ÍTEMS $25,26,27,28,29,30,31,32,33,34,35$ у 36 \\
\hline ÍTEM 31 & ÍTEMS $10,12,14,24,25,26,27,28,29,30,31,32,33,34,35$ у 36 \\
\hline ÍTEM 32 & ÍTEMS $12,14,24,25,26,27,28,29,30,31,32,33,34,35$ y 36 \\
\hline ITTEM 33 & ÍTEMS $14,25,26,27,28,29,30,31,32,33,34,35$ у 36 \\
\hline ITTEM 34 & ÍTEMS 12, 14, 25, 26, 27, 28, 29, 30, 31, 32, 33, 34, 35 у 36 \\
\hline ITTEM 35 & İTEMS 12, 14, 24, 25, 26, 27, 28, 29, 30, 31, 32, 33, 34, 35 у 36 \\
\hline ITTEM 36 & ÍTEMS 10, 12, 14, 24, 25, 26, 27, 28, 29, 30, 31, 32, 33, 34, 35 у 36 \\
\hline
\end{tabular}

En la Tabla 2 se detallan los índices de discriminación de cada ítem respecto al total. Ningún valor es $<0,2$ por lo que ningún ítem es problemático y no es necesario eliminarlo.

Tabla 2. Nivel de discriminación por ítem respecto al total

\begin{tabular}{|c|c|c|c|}
\hline ÍTEM & ÍNDICE DE DISCRIMINACIÓN & ÍTEM & ÍNDICE DE DISCRIMINACIÓN \\
\hline ITEM 10 & 0,533 & ÍTEM 24 & 0,518 \\
\hline ITEM 11 & 0,279 & ÍTEM 25 & 0,598 \\
\hline ITEM 12 & 0,631 & ITTEM 26 & 0,667 \\
\hline ÍTEM 13 & 0,472 & ÍTEM 27 & 0,593 \\
\hline ITEM 14 & 0,606 & ITTEM 28 & 0,668 \\
\hline ÍTEM 15 & 0,250 & ÍTEM 29 & 0,699 \\
\hline ÍTEM 16 & 0,379 & ÍTEM 30 & 0,627 \\
\hline ITEM 17 & 0,327 & ÍTEM 31 & 0,739 \\
\hline ÍTEM 18 & 0,437 & ÍTEM 32 & 0,766 \\
\hline ITEM 19 & 0,297 & ÍTEM 33 & 0,641 \\
\hline ÍTEM 20 & 0,411 & ITEM 34 & 0,716 \\
\hline ÍTEM 21 & 0,338 & ÍTEM 35 & 0,670 \\
\hline ÍTEM 22 & 0,426 & ITEM 36 & 0,753 \\
\hline ITTEM 23 & 0,426 & & \\
\hline
\end{tabular}

Para relacionar los ítems dicotómicos se ha procedido a realizar la prueba chi cuadrado de Pearson y se destacan aquellas relaciones que son más significativas. 


\section{Relación "Rol académico" e "ÍTEM 8"}

Figura 14. Tabla de contingencia "Rol académico" e "ÍTEM 8"

\begin{tabular}{|c|c|c|c|c|c|}
\hline & & & \multicolumn{2}{|c|}{$\begin{array}{c}\text { ¿Conoce en que consiste la } \\
\text { gamificación? }\end{array}$} & \multirow[b]{2}{*}{ Total } \\
\hline & & & Si & No & \\
\hline \multirow[t]{3}{*}{ Rol académico actual } & Estudiante & Recuento & 30 & 39 & 69 \\
\hline & & $\begin{array}{l}\text { \% dentro de Rol } \\
\text { académico actual }\end{array}$ & $43,5 \%$ & $56,5 \%$ & $100,0 \%$ \\
\hline & Docente & $\begin{array}{l}\text { Recuento } \\
\text { \% dentro de Rol } \\
\text { académico actual }\end{array}$ & $\begin{array}{r}17 \\
77,3 \%\end{array}$ & $\begin{array}{r}5 \\
22,7 \%\end{array}$ & $\begin{array}{r}22 \\
100,0 \%\end{array}$ \\
\hline Total & & $\begin{array}{l}\text { Recuento } \\
\text { \% dentro de Rol } \\
\text { académico actual }\end{array}$ & $\begin{array}{r}47 \\
51,6 \%\end{array}$ & $\begin{array}{r}44 \\
48,4 \%\end{array}$ & $\begin{array}{r}91 \\
100,0 \%\end{array}$ \\
\hline
\end{tabular}

Figura 15. Tabla prueba chi-cuadrado "Rol académico" e "ÍTEM 8"

\begin{tabular}{|c|c|c|c|c|c|}
\hline \multicolumn{6}{|c|}{ Pruebas de chi-cuadrado } \\
\hline & Valor & $\mathrm{gl}$ & $\begin{array}{c}\text { Sig. asintótica } \\
\text { (bilateral) }\end{array}$ & $\begin{array}{c}\text { Sig. exacta } \\
\text { (bilateral) }\end{array}$ & $\begin{array}{l}\text { Sig. exacta } \\
\text { (unilateral) }\end{array}$ \\
\hline Chi-cuadrado de Pearson & $7,629^{a}$ & 1 &, 006 & & \\
\hline $\begin{array}{l}\text { Corrección por } \\
\text { continuidad }\end{array}$ & 6,336 & 1 & 012 & & \\
\hline Razón de verosimilitudes & 7,995 & 1 &, 005 & & \\
\hline $\begin{array}{l}\text { Estadístico exacto de } \\
\text { Fisher }\end{array}$ & & & &, 007 & ,005 \\
\hline $\begin{array}{l}\text { Asociación lineal por } \\
\text { lineal }\end{array}$ & 7,545 & 1 &, 006 & & \\
\hline N de casos válidos & 91 & & & & \\
\hline
\end{tabular}

Se ha querido conocer el grado de relación entre el rol académico y "¿Conoce en qué consiste la gamificación?", como se puede observar tienen un valor $\mathrm{p}$ significativo $(0,006<0,05)$, por lo tanto, si existe relación entre estos ítems. Se puede interpretar como que de aquellos que conocen en qué consiste la gamificación, en su mayoría, son docentes y de los estudiantes participantes su mayoría no conocían antes de este estudio de que trataba esta metodología.

\section{Relación "Intervalo de edad" e "ÍTEM 9"}

Figura 16. Tabla de contingencia "Intervalo de edad" e "ÍTEM 9"

\begin{tabular}{|c|c|c|c|c|c|}
\hline & & & \multicolumn{2}{|c|}{$\begin{array}{l}\text { ¿Considera que la } \\
\text { gamificación puede ser una } \\
\text { propuesta interesante para } \\
\text { implementarla en el ámbito de } \\
\text { la formación e-learning? }\end{array}$} & \multirow[b]{2}{*}{ Total } \\
\hline & & & si & No & \\
\hline \multirow[t]{4}{*}{$\begin{array}{l}\text { Señala el rango de edad } \\
\text { al que usted pertenece }\end{array}$} & De 16 a 25 aก̃os & $\begin{array}{l}\text { Recuento } \\
\text { \% dentro de Señala el } \\
\text { rango de edad al que } \\
\text { usted pertenece }\end{array}$ & $\begin{array}{r}58 \\
96,796\end{array}$ & $\begin{array}{r}2 \\
3,3 \%\end{array}$ & $\begin{array}{r}60 \\
100,0 \%\end{array}$ \\
\hline & De 26 a 35 años & $\begin{array}{l}\text { Recuento } \\
\text { \% dentro de Señala el } \\
\text { rango de edad al que } \\
\text { usted pertenece }\end{array}$ & $\begin{array}{r}17 \\
81.0 \%\end{array}$ & $\begin{array}{r}4 \\
19.0 \%\end{array}$ & $\begin{array}{r}21 \\
100,096\end{array}$ \\
\hline & De 36 a 45 años & $\begin{array}{l}\text { Recuento } \\
\text { \% dentro de Señala el } \\
\text { rango de edad al que } \\
\text { usted pertenece }\end{array}$ & $\begin{array}{r}14 \\
100,0 \%\end{array}$ & $\begin{array}{r}0 \\
0,0 \%\end{array}$ & $\begin{array}{r}14 \\
100,0 \%\end{array}$ \\
\hline & Mayores de 55 años & $\begin{array}{l}\text { Recuento } \\
\text { \% dentro de Señala el } \\
\text { rango de edad al que } \\
\text { usted pertenece }\end{array}$ & $\begin{array}{r}5 \\
100,0 \%\end{array}$ & $\begin{array}{r}0 \\
0,0 \%\end{array}$ & $\begin{array}{r}5 \\
100,096\end{array}$ \\
\hline Total & & $\begin{array}{l}\text { Recuento } \\
\% \text { dentro de Señala el } \\
\text { rango de edad al que } \\
\text { usted pertenece }\end{array}$ & $\begin{array}{r}94 \\
94,096\end{array}$ & $\begin{array}{r}6 \\
6,096\end{array}$ & $\begin{array}{r}100 \\
100,0 \%\end{array}$ \\
\hline
\end{tabular}


Figura 17. Tabla prueba chi-cuadrado "Intervalo de edad" e "ÍTEM 9"

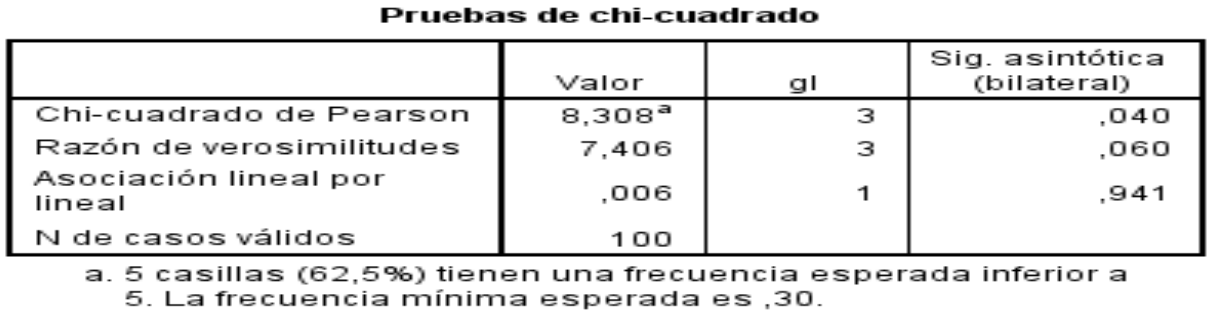

Como se puede observar, el valor de la significación es de 0,040 y por tanto es menor que 0,05 , por lo que existe relación entre el ítem "Intervalo de edad" y el ítem "¿Considera que la gamificación puede ser una propuesta interesante para implementarla al ámbito de formación e-learning?". Lo que significa, teniendo también en cuenta los datos de la figura 89 , que la totalidad de los sujetos que pertenecen a los dos últimos grupos de edad consideran la gamificación una opción para incorporar a la teleformación. De los rangos de edad mayoritarios, del intervalo de 16 a 25 años solo un $3 \%$ no lo considera una alternativa interesante y de los 21 sujetos de edades entre 26 y 35 años, únicamente 4 no lo ven como una posibilidad llamativa para el ámbito propuesto.

Relación "ÍTEM 8" e "ÍTEM 9"

Figura 18. Tabla de contingencia "ÍTEM 8" e "ÍTEM 9"

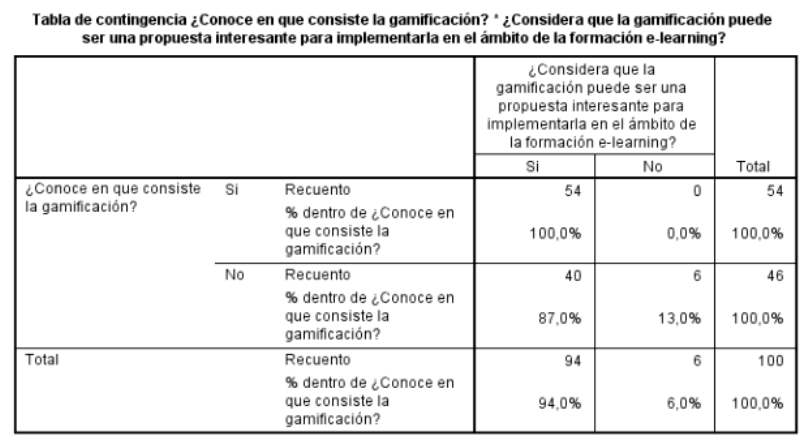

Figura 19. Tabla prueba chi-cuadrado "ÍTEM 8" e "ÍTEM 9"

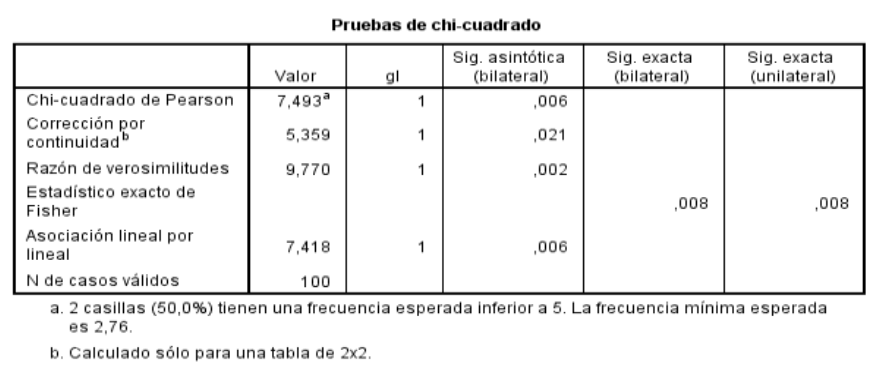

En este caso se ha querido determinar el grado de correlación entre la cuestión “¿Conoce en qué consiste la gamificación?" y “¿Considera que la gamificación puede ser una propuesta interesante para implementarla en el ámbito de la formación elearning?". El resultado ha sido que, si existe un alto nivel de relación entre estos ítems, ya que como podemos ver en la Figura 94, la significación asintónica bilateral es de 0,006 y por tanto es bastante menor que 0,05. Esto se traduce en que aquellos sujetos que conocen de que trata la gamificación también lo ven como una propuesta 
interesante para introducirlo en el ámbito de la formación e-learning. Y aquellos que no conocían en qué consiste, tras una pequeña definición, también lo han considerado como una alternativa atractiva.

\section{C) Análisis e interpretación de los ítems mediante ANOVA}

Intervalo edad e ítems escala likert.

En la siguiente tabla (figura 20) se relaciona el grupo etario con los ítems de tipo Likert, se procederá para tener en cuenta aquellos que sean significativos, es decir que tengan un valor $\mathrm{P}<0,05$.

Figura 20. Tabla ANOVA "Intervalo edad"

\begin{tabular}{|c|c|c|c|c|c|c|}
\hline & & $\begin{array}{l}\text { Suma de } \\
\text { cuadrados }\end{array}$ & $g$ & $\begin{array}{c}\text { Media } \\
\text { cuadratica }\end{array}$ & $\mathrm{F}$ & Sig \\
\hline 10. Levarar a cabo cursos a través de Internet (oniline) me offece más benefficios que desventajas & $\begin{array}{l}\text { Inter-grupos } \\
\text { Intra-grupos }\end{array}$ & $\begin{array}{r}6,6600 \\
54,090\end{array}$ & \begin{tabular}{r|r|}
3 \\
96
\end{tabular} & $\begin{array}{r}2.220 \\
.563\end{array}$ & 3.940 & .011 \\
\hline 11. Las tecnologias de la intormación unllizadas en formación e-learning son fácilles de usar & $\begin{array}{l}\text { Total } \\
\text { Inter-gupos } \\
\text { Intra-gupoos }\end{array}$ & \begin{tabular}{r|}
600,750 \\
3,683 \\
42,907
\end{tabular} & \begin{tabular}{r|}
99 \\
3 \\
96
\end{tabular} & $\begin{array}{r}1.228 \\
.447\end{array}$ & 2,747 & .047 \\
\hline & & 46,590 & 99 & & & \\
\hline 12. Las tecnologías de la información ullilizadas en tormación e-learming ofrecen tunciones útles que facilitan el aprendizaje & $\begin{array}{l}\text { Inter-gupos } \\
\text { Intra-guvpos } \\
\text { Total }\end{array}$ & $\begin{array}{r}2,140 \\
41,250 \\
43,390\end{array}$ & \begin{tabular}{r|r|}
3 \\
96 \\
99
\end{tabular} & $\begin{array}{l}.713 \\
.430\end{array}$ & 1.660 & .181 \\
\hline 13. La comunicación que se da a través de la tormación eleleaming es adecuada y de buena callidad & $\begin{array}{l}\text { Inter-grupos } \\
\text { Intragupos } \\
\text { Total }\end{array}$ & $\begin{array}{r}2,010 \\
49,950 \\
51,960\end{array}$ & \begin{tabular}{l|l|} 
& 3 \\
96 & \\
99 & \\
\end{tabular} & $\begin{array}{l}.670 \\
.520\end{array}$ & 1,288 & .283 \\
\hline 14.El uso de platatormas web aumenta el dasemperío yefectividad respecto a la raalizaclín de tareas & $\begin{array}{l}\text { Inter-grupos } \\
\text { Intragupos } \\
\text { Total }\end{array}$ & \begin{tabular}{|l|l|}
1,554 \\
66,086 \\
67,640
\end{tabular} & \begin{tabular}{|l|l|} 
& 3 \\
96 & \\
99 & \\
\end{tabular} & $\begin{array}{l}.518 \\
.688 \\
\end{array}$ & .753 & .523 \\
\hline 15. Considero sencillo o I manejo de platatormas web & $\begin{array}{l}\text { Inter-grupos } \\
\text { Intra-grupos } \\
\text { Totalal }\end{array}$ & \begin{tabular}{|r|}
7,188 \\
43,402 \\
50,590
\end{tabular} & \begin{tabular}{r|r}
3 \\
96 \\
99
\end{tabular} & $\begin{array}{r}2,396 \\
452\end{array}$ & 5.299 & .002 \\
\hline 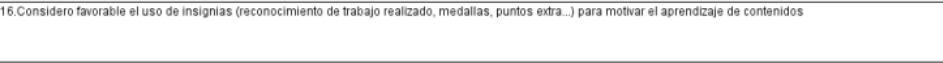 & $\begin{array}{l}\text { Inter-grupos } \\
\text { Intra-gupos } \\
\text { Total }\end{array}$ & $\begin{array}{r}2,959 \\
42,831 \\
45,790 \\
4\end{array}$ & \begin{tabular}{l|l|}
3 & \\
96 & \\
99 & \\
\end{tabular} & $\begin{array}{l}.986 \\
.446\end{array}$ & 2,211 & .092 \\
\hline 17. Considero positivo el uso de insignias para mejorar la compentividad sana entre compañeros & $\begin{array}{l}\text { Inter-grupos } \\
\text { Intra-gupos } \\
\text { Total }\end{array}$ & \begin{tabular}{r|}
3,474 \\
75,686 \\
79,160
\end{tabular} & \begin{tabular}{r|r|}
3 & \\
96 & \\
99 &
\end{tabular} & $\begin{array}{r}1.158 \\
, 788\end{array}$ & 1.469 & .228 \\
\hline 18. Considero que los recursos didá́cticos (textos, videos, Juegos.) permiten avanzar a un nitmo propio de aprendizale & $\begin{array}{l}\text { Inter-grupos } \\
\text { Intra-grupos } \\
\text { Total } \\
\end{array}$ & \begin{tabular}{|r|}
2,261 \\
34,579 \\
36,840 \\
3
\end{tabular} & \begin{tabular}{r|r}
3 \\
96 \\
99 \\
\end{tabular} & $\begin{array}{l}.754 \\
.360\end{array}$ & 2,093 & .106 \\
\hline 19.Considero que el contenido de videos explicativos con ejercicicos desarrollados paso a paso permiten mejorar la comprensión de un tema & $\begin{array}{l}\text { Inter-gupos } \\
\text { Intra-grupos } \\
\text { Total }\end{array}$ & $\begin{array}{r}1,554 \\
33,436 \\
34,990\end{array}$ & \begin{tabular}{r|r}
3 \\
96 \\
99
\end{tabular} & $\begin{array}{l}.518 \\
.348 \\
\end{array}$ & 1.488 & .223 \\
\hline 20.Considero que el desartollo de actividades en grupo como "retoss" mejoran el aprendizaje de un tema & $\begin{array}{l}\text { Inter-grupos } \\
\text { Intrag-gupos } \\
\text { Total } \\
\end{array}$ & $\begin{array}{r}3.460 \\
34,900 \\
38,360 \\
\end{array}$ & \begin{tabular}{r|r}
3 & \\
96 & \\
99 & \\
\end{tabular} & $\begin{array}{r}1.153 \\
.364\end{array}$ & 3.172 & .028 \\
\hline 21. Considero que actividades lǘlicas (crucigramas, sopas de letras.) permiten reftrarar los conocimientos sobre un tema & $\begin{array}{l}\text { Inter-grupos } \\
\text { Intra-gupos } \\
\text { Total }\end{array}$ & \begin{tabular}{|r|}
1,420 \\
54,140 \\
55,560
\end{tabular} & \begin{tabular}{r|r}
3 & \\
96 & \\
99 & \\
\end{tabular} & $\begin{array}{l}.473 \\
.564\end{array}$ & 839 & .476 \\
\hline 22. Considero que el empleo de dispos stituos móviles (ordenadores, móviles, pads.) pueden ayudar en el processo de aprendizale de temas & $\begin{array}{l}\text { Inter-grupos } \\
\text { Intra-gupos } \\
\text { Total }\end{array}$ & \begin{tabular}{|r|}
1,923 \\
39,867 \\
41,790 \\
\end{tabular} & \begin{tabular}{r|r}
3 & \\
96 & \\
99 &
\end{tabular} & $\begin{array}{l}.641 \\
.415\end{array}$ & 1.544 & .208 \\
\hline 23. Considero que la realzzación de diferentes tipos cuestionarios para poner a prueba los conocimientos de un tema antes de un examen son tavorables para el aprendzaje & $\begin{array}{l}\text { Intergrupos } \\
\text { Intra-grupos } \\
\text { Total } \\
\end{array}$ & $\begin{array}{r}1,339 \\
35,571 \\
36,910 \\
\end{array}$ & \begin{tabular}{r|r}
3 \\
96 \\
99
\end{tabular} & $\begin{array}{l}.446 \\
.371\end{array}$ & 1.204 & .312 \\
\hline 24. Considero postivo poder colaborar de torma activa en mi evaluación y en la de ottros compañeros & $\begin{array}{l}\text { Inter-grupos } \\
\text { Intraguppos } \\
\text { Total }\end{array}$ & $\begin{array}{r}2,580 \\
55,860 \\
58,440\end{array}$ & \begin{tabular}{|l|l|}
9 & \\
96 & \\
99 & \\
\end{tabular} & $\begin{array}{l}.860 \\
.582\end{array}$ & 1.478 & .225 \\
\hline 25. Difundir notificaciones en entornos virtuales de formación & $\begin{array}{l}\text { Inter-grupos } \\
\text { Intra-gupos } \\
\text { Total }\end{array}$ & $\begin{array}{r}1,561 \\
44,979 \\
46,560\end{array}$ & \begin{tabular}{r|r}
3 \\
96 \\
99
\end{tabular} & $\begin{array}{l}.527 \\
.469\end{array}$ & 1.125 & .343 \\
\hline 26. Publicar intormación en blogs, pảginas webs o platatormas educativas de teleftormación (como Moodile) & $\begin{array}{l}\text { Inter-guvos } \\
\text { Intraguvos } \\
\text { Total } \\
\end{array}$ & $\begin{array}{r}9.952 \\
51,638 \\
52,590 \\
5\end{array}$ & \begin{tabular}{r|}
3 \\
96 \\
99 \\
\end{tabular} & $\begin{array}{l}.317 \\
.538 \\
\end{array}$ & .590 & .623 \\
\hline 27. Gestionary edtar imágenes en formato digtal & $\begin{array}{l}\text { Inter-grupos } \\
\text { Intra-guvoos } \\
\text { Total }\end{array}$ & $\begin{array}{r}1,624 \\
59,376 \\
61,000 \\
\end{array}$ & \begin{tabular}{c|c}
3 \\
96 \\
99 \\
\end{tabular} & $\begin{array}{l}.541 \\
.619\end{array}$ & .875 & .457 \\
\hline 28. Elaborary y editar archivos de sonido y audio en formato digital & $\begin{array}{l}\text { Inter-grupos } \\
\text { Intra-gupos } \\
\text { Total }\end{array}$ & $\begin{array}{r}\quad 711 \\
71,079 \\
71,790\end{array}$ & \begin{tabular}{l|l}
3 \\
96 \\
99 \\
\end{tabular} & $\begin{array}{l}.237 \\
.740\end{array}$ & 320 & 811 \\
\hline 29.Edtary y manipular videos digitales para la creación de materiales audiovisuales & $\begin{array}{l}\text { Inter-grupos } \\
\text { Intra-gupos } \\
\text { Total }\end{array}$ & \begin{tabular}{r|}
2,081 \\
74,279 \\
76,360 \\
\end{tabular} & \begin{tabular}{|l|}
3 \\
96 \\
99 \\
\end{tabular} & $\begin{array}{l}.694 \\
.774\end{array}$ & .897 & .446 \\
\hline 30.Elaborar animaciones a través del ordenador, con o sin interactividad & $\begin{array}{l}\text { Inter-grupos } \\
\text { Intra-grupos } \\
\text { Total } \\
\end{array}$ & $\begin{array}{r}1,979 \\
98,771 \\
100,750 \\
\end{array}$ & \begin{tabular}{|r|}
3 \\
96 \\
99 \\
\end{tabular} & $\begin{array}{r}.660 \\
1.029\end{array}$ & .641 & .590 \\
\hline 31. Elaborar materiales didácticos centrados en el aprendizaje, basados en la realización de actividades integradas en los contenidos que se quieren desarrollar & $\begin{array}{l}\text { Inter-grupos } \\
\text { Intra-grupos } \\
\text { Total }\end{array}$ & $\begin{array}{r}414 \\
57,586 \\
58,000\end{array}$ & \begin{tabular}{r|}
3 \\
96 \\
99 \\
\end{tabular} & $\begin{array}{l}.138 \\
.600\end{array}$ & 230 & .875 \\
\hline 32.Elaborar materiales didácticos centrados en el aprendizaje y dotados de interactividad & $\begin{array}{l}\text { Inter-grupos } \\
\text { Intra-gupos } \\
\text { Total }\end{array}$ & $\begin{array}{r}677 \\
65,033 \\
65,710 \\
\end{array}$ & \begin{tabular}{l|l}
3 \\
96 \\
99 \\
9
\end{tabular} & $\begin{array}{l}.226 \\
.677\end{array}$ & 333 & .802 \\
\hline 33.Elaborara actividades de desarrollo temätico multimedia & $\begin{array}{l}\text { Inter-guvos } \\
\text { Intra-grupos } \\
\text { Total }\end{array}$ & $\begin{array}{r}2,392 \\
57,848 \\
60,240 \\
1527\end{array}$ & \begin{tabular}{r|}
3 \\
96 \\
99 \\
\end{tabular} & . .797 & 1,323 & .271 \\
\hline 34.Plantear actividades mediante foros de discusión en un entormo virtual de formación (Moodle) & $\begin{array}{l}\text { Inter-gupos } \\
\text { Intra-gunpos } \\
\text { Total }\end{array}$ & $\begin{array}{r}1,527 \\
62,183 \\
63,710 \\
\end{array}$ & \begin{tabular}{r|}
3 \\
96 \\
99 \\
\end{tabular} & $\begin{array}{l}.509 \\
.648\end{array}$ & .786 & .505 \\
\hline 35. Plantear diálogos o debates en linea mediante audiohideoconferencia & $\begin{array}{l}\text { Inter-grupos } \\
\text { Intra-gupos } \\
\text { Total }\end{array}$ & $\begin{array}{r}2,093 \\
54,907 \\
57,000\end{array}$ & \begin{tabular}{l|l}
3 \\
96 \\
99
\end{tabular} & $\begin{array}{l}.698 \\
.572 \\
\end{array}$ & 1.220 & .307 \\
\hline 36. Elaborar actividades con carácter comunicativo mediante las redes sociales & $\begin{array}{l}\text { Inter-grupos } \\
\text { Intragugupos } \\
\text { Total }\end{array}$ & \begin{tabular}{|l|l|}
1,081 \\
79,669 \\
80,750 \\
\end{tabular} & \begin{tabular}{l|}
3 \\
96 \\
99 \\
\end{tabular} & $\begin{array}{l}.360 \\
.830\end{array}$ & .434 & .729 \\
\hline
\end{tabular}


Se pueden destacar los ítems 10 (p: 0,011), 11 (p: 0,042), 15 (p: 0,002) y $20(0,026)$, para que se pueda contemplar de una manera más clara, se presentan los gráficos donde se comparan las medias de los ítems 10, 11, 15 y 20 con el intervalo de edad:

Figura 21. Gráficos ANOVA “Intervalo de edad"
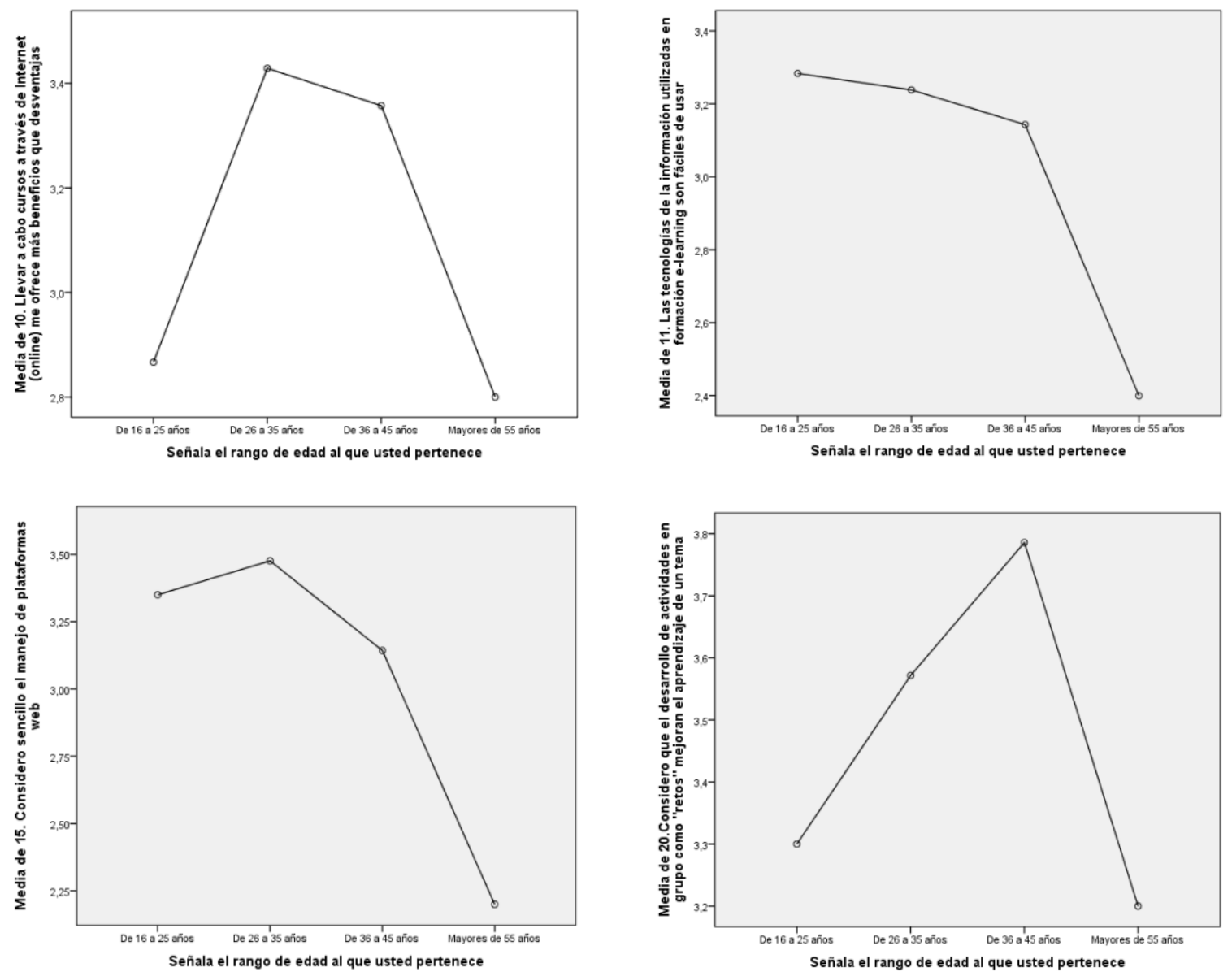

Se puede observar cómo, algo que tienen en común estos gráficos, al igual que otros que no tienen diferencias significativas, pero tienen un valor $p$ también bajo, es que en el último intervalo de edad, es decir, en aquellos participantes mayores de 55 años, la media baja muy exponencialmente, se puede entender como que aquellas personas que son más mayores presentan más necesidades y no tienen la misma facilidad para adaptarse a las nuevas realidades tecnológicas.

\section{Rol académico y escala likert}

Se sigue el mismo procedimiento anterior pero esta vez relacionando el "rol académico" con los ítems de escala likert. A partir de la tabla que se genera, se seleccionan y aquí se señalan aquellos que han sido significativos: ítem 10 (p: 0,029), ítem 20 (p: 0,002), ítem 33 (p: 0,003).

A partir de las gráficas se puede interpretar como los docentes consideran más favorable llevar a cabo cursos a través de internet y de forma general, la utilización de recursos relacionados con la gamificación. Por otro lado, los estudiantes ven a los docentes mejor preparados y capacitados para el manejo de materiales didácticos digitales. 
Se procede a analizar el mismo conjunto de ítems con el ítem 8 , ¿Conoce en qué consiste la gamificación?", en esta ocasión han surgido bastantes valores significativos en los siguientes ítems: ítem 10 (p: 0,001), ítem 12 (p: 0,027), ítem 13 (p: 0,024), ítem 14 (p: 0,000), ítem 16 (p: 0,003), ítem 17 (p: 0,003), ítem 18: 0,007), ítem 20 (p: 0,041) e ítem $21(0,001)$.

Como interpretación, se puede destacar que aquellos participantes que conocían la gamificación consideran favorable el uso de elementos de la gamificación y la teleformación, ya que donde los participantes conocen la gamificación es más alta la media del ítem.

\section{Ítem 9 y escala likert}

Se relacionan los ítems de escala likert con la cuestión "¿Consideras que la gamificación puede ser una propuesta interesante para implementarla en el ámbito de la formación e-learning?", tras la observación de la tabla que se produce destacamos los valores significativos de los siguientes ítems: ítem 11 (p: 0,045), ítem 16 (p: 0,038), ítem 19 (p: 0,037), ítem 21 (p: 0,015) e ítem 22 (p: 0,004).

Analizando las gráficas correspondientes, se puede señalar que esta relación tiene sentido, los sujetos que han considerado como buena opción la gamificación para implementarla en la formación e-learning están de acuerdo con la utilización de elementos clave y fundamentales de esta metodología.

\section{Discusión}

Tras tener una primera visión de la bibliografía respecto a las investigaciones sobre la formación, la teleformación y la gamificación y sobre su situación actual, y tras la información recogida a través del cuestionario y su correspondiente interpretación, se ha llegado a las siguientes conclusiones respecto a los objetivos previamente planteados:

Atendiendo al "OE1 Conocer el grado de conocimiento sobre gamificación y el manejo en TIC según el género, edad, rol académico y nivel educativo" se puede afirmar tras interpretar los datos del estudio que respecto a la edad, aquellos que presentan mayores necesidades en el manejo de las TIC son el profesorado femenino mayor de 55 años y respecto a la gamificación presentan más desconocimiento los estudiantes. No se puede especificar la edad y el nivel educativo porque no ha salido que esa combinación tenga significación y por lo tanto, tampoco relación.

Los estudiantes en un principio no conocen esta metodología, pero si la consideran útil tras una breve explicación sobre su finalidad y esto se ve reflejado en las respuestas recogidas en el ítem "¿Considera que la gamificación puede ser una propuesta interesante para implementarla en el ámbito de la formación e-learning?". De esta manera se responde al "OE3 Observar en qué medida la gamificación se considera una buena metodología cuando se tiene conocimiento sobre su finalidad" al cuál se responde con que, tanto profesorado como alumnado perteneciente a cualquier intervalo de edad considera la gamificación una buena propuesta. Quien conoce las bases de la gamificación lo considera una buena alternativa para incluirla en cualquier tipo de formación. 
Esta conclusión también se ve alcanzada a través de las respuestas a los ítems relacionados con elementos de la gamificación, ya que se ha comprobado cómo son los ítems mejor valorados, por lo que se cumple "OE4: Conocer el grado de utilidad que consideran que tienen los principales elementos de la gamificación" por el que además se puede confirmar como el grado de utilidad considerado es alto.

Respecto al "OE2: Conocer cuáles son las plataformas web más utilizadas según los diferentes roles educativos" no hay correlación entre estos ítems, pero se puede percibir como, tras los datos recogidos, la plataforma web más utilizada por estudiantes es Moodle y el profesorado utiliza con más frecuencia Microsoft Teams.

Por otro lado, se puede afirmar que el objetivo "OE5: Conocer el nivel de conocimiento y capacitación que tiene el profesorado respecto al manejo de entornos virtuales y recursos digitales" se ha cumplido y se puede afirmar como se encuentran bastantes necesidades y cabe destacar como los estudiantes ven mejor preparados a los docentes en este aspecto que el propio profesorado.

Asimismo, atendiendo al objetivo general, "OG1: Recoger información a través de un cuestionario sobre la percepción que tiene el profesorado y alumnado respecto al uso de gamificación en formación e-learning y detectar posibles necesidades para poder llevarlo a cabo" se puede concluir afirmando que se ha recogido información suficiente como para determinar que las percepciones y predisposiciones tanto de alumnado como de profesorado son positivas ante la implementación de gamificación en formación e-learning, pero que se encuentran ciertas limitaciones y necesidades de formación digital en el profesorado para organizar y crear materiales y recursos didácticos digitales, centrados en el aprendizaje que se quiere alcanzar, y ricos en interactividad y motivación, aspectos que son fundamentales en la gamificación.

Referencias

Arias, J., Contreras, J.A., Espada, R. M. \& Melo, M. (2017). Validación de un cuestionario de satisfacción para la introducción de la gamificación móvil en la educación superior. Revista Ibérica de Sistemas y Tecnologías de Información, 23, 33-45. Doi: 10.17013/risti.23.33-45

Covas, V. (2020). Estudio sobre el uso de la gamificación en plataformas de e-learning: teorías de comportamiento, tasas de participación y experiencias de uso. Sintaxis, 1(5), 128147. https://doi.org/https://doi.org/10.36105/stx.2020n5.07

Ebel, R. L. (1965). Measuring educational achievement. Englewood: Prentice-Hall.

Expósito Unday, D., \& González Valero, J. A. (2017) Sistematización de experiencias como método de investigación. Gaceta Médica Espirituana, 19(2), 1-6.

Francés, J., Bleda, S., Calzado, E. M., Martínez, F.J., Heredia, S., Hernández, A., Hidalgo, A., Vera, J. \& Yebra, M. S. (2019). Análisis y aplicación de nuevas metodologías docentes basadas en clase invertida y gamificación a través de Moodle.

Herrera-Sánchez, D., Padereweski-Rodríguez, P. \& Gutiérrez, F. L. (2015). U-Learning Gamification: Gamificación aplicada a entornos ubicuos de enseñanza y aprendizaje. Congreso Iberoamericano de Ambientes de Aprendizaje Futuros (CIAAF'15), 12.
Ortiz-Colón, A. M., Jordán, J. \& Agredal, M. (2018). Gamificación en educación: una panorámica sobre el estado de la cuestión. Educação e Pesquisa, 44, 1-17. Doi: http://dx.doi.org/10.1590/s1678-4634201844173773

Padial, J.J. \& Berrocal, E. (2021). Conocimiento del Medio y Diversidad. Aproximación al interés de alumnos con dificultades de integración hacia las Ciencias Sociales. REIDOCREA, 10(8), 1-18.

Romero, J.J., Moreno, A., \& Sola, T. (2012). Estudio de necesidades de formación de los profesores andaluces en el ámbito de la autoría de materiales educativos digitales en ambientes virtuales de aprendizaje. Journal for Educators, Teachers and Trainers, 3(1), 92-108.

Zambrano, J. (2016). Factores predictores de la satisfacción de estudiantes de cursos virtuales. Revista Iberoamericana de Educación a Distancia, 19(2), 217-235. Doi: http://dx.doi.org/10.5944/ried.19.2.15112 